\title{
Revascularização no Pé Diabético: Caso Clínico
}

\section{Diabetic Foot Revascularization: Case Report}

Ana Pinheiro¹, Daniel Brandão², Paulo Barreto², Joana Ferreira², José Almeida Lopes², Joel Sousa², Armando Mansilha²

\section{RESUMO}

Em Portugal, a prevalência da diabetes mellitus é cerca de 13,3\%. Uma das complicações da diabetes é a amputação. Destas, 70\% são precedidas de uma úlcera.

Apresentamos o caso de um doente do sexo masculino de 46 anos de idade, fumador, com antecedentes de diabetes mellitus não controlada, que recorreu ao serviço de urgência por uma úlcera no pé direito com cerca de dois meses de evolução. Foi internado com o diagnóstico de pé diabético neuroisquémico, para controlo de infeção, analgesia e revascularização.

Consideramos o caso clínico pertinente dada a prevalência crescente de diabetes mellitus e da doença arterial obstrutiva periférica. Salienta-se a importância da instituição de tratamento precoce, em particular da revascularização e do controlo da infeção, o que conduzirá à diminuição da taxa de amputações major.

PALAVRAS-CHAVE: Amputação; Complicações da Diabetes; Doença Arterial Obstrutiva; Isquemia; Pé Diabético; Procedimentos Endovasculares 


\begin{abstract}
In Portugal, the prevalence of diabetes mellitus is about 13.3\%. One of the major complications of diabetes is the amputation, $70 \%$ of these are preceded of an ulcer.

This case is about a 46-year-old patient, smoker, with history of uncontrolled diabetes mellitus that went to the emergency department with an ulcer on the right foot with about two months of evolution. He was hospitalized with the diagnosis of diabetic neuroischemic foot, for control of infection, analgesia and revascularization.

We consider this case report relevant due to the growing prevalence of diabetes mellitus and peripheral obstructive arterial disease. It is important to emphasize the importance of early treatment, especially revascularization and infection control, that will lead to a reduction in the rate of major amputations.
\end{abstract}

KEYWORDS: Amputation; Complications of Diabetes; Diabetic Foot; Endovascular Procedures; Ischemia; Peripheral Arterial Disease

\section{INTRODUÇÃO}

Atualmente, em Portugal, a prevalência da diabetes mellitus (DM) é cerca de 13,3\%, sendo que destes, apenas 7,5\% têm a sua doença diagnosticada. ${ }^{1}$ A DM constitui uma das principais causas de morte, principalmente por implicar um risco significativamente aumentado de doença coronária e de acidente vascular cerebral. Segundo o Instituto Nacional de Estatística, em 2015 morreram 4406 pessoas com causa diretamente relacionada com a DM. ${ }^{2}$ A persistência de um nível de hiperglicemia, mesmo quando não se verificam os sintomas para alertar o indivíduo para a presença de doença ou para a sua descompensação, resulta em múltiplas lesões mais marcadas ao nível dos rins, olhos, nervos periféricos e sistema vascular. ${ }^{3}$

Entre 40\% a 70\% de todas as amputações das extremidades inferiores são devidas ao pé diabético e precedidas de uma ulceração no pé. Os fatores mais importantes relacionados com o desenvolvimento de úlceras são a neuropatia periférica, traumas superficiais e deformidades no pé. ${ }^{4}$ Existem duas patologias no pé diabético: a polineuropatia distal que origina o pé neuropático e a oclusão arterial que dá origem ao pé neuroisquémico. A úlcera isquémica tem uma localização diferente de uma úlcera neuropática, localizando-se mais frequentemente nas margens do pé, incluindo as extremidades do dedo e calcanhar tal como no caso clínico descrito.

Uma das grandes associações com a DM é a doença arterial obstrutiva periférica (DAOP). A sua apresentação clínica engloba a claudicação intermitente, a dor em repouso e a ulceração com ou sem gangrena. ${ }^{5}$ A DAOP leva a uma duplicação do risco de infeção. A isquemia no pé diabético vai comprometer o fluxo sanguíneo, causando diminuição da resposta inflamatória e imunitária à infeção e a um menor aporte local da antibioterapia. Nestes doentes o atingimento arterial é preferencialmente infragenicular, cursando frequentemente a este nível com lesões mais complexas. O tratamento endovascular das artérias infrageniculares está descrito como uma forma terapêutica eficaz em doentes com isquemia crítica e atingimento deste setor. Com o desenvolvimento da tecnologia endovascular tornou-se possível o tratamento de segmentos arteriais distais dos membros, através da utilização de guias, cateteres, balões e stents de baixo perfil. ${ }^{6}$

Nos pacientes com DM, qualquer infeção nos pés é potencialmente séria. Estas envolvem mais comummente os tecidos moles, podendo em um quinto dos casos estender-se para o osso. A prevenção e o diagnóstico imediato são necessários para prevenir a morbilidade, especialmente a decorrente da amputação. A infeção raramente cede, sem a ação sinérgica da cirurgia com a antibioterapia.

\section{CASO CLÍNICO}

Doente do sexo masculino de 46 anos de idade, fumador (20 UMA), com antecedentes de DM não controlada $(\mathrm{HbA} 1 \mathrm{c}=10 \%)$, retinopatia e hipertensão arterial. Medicado cronicamente com ácido acetilsalicílico 150 mg (uma vez ao dia), metformina 1 g (três vezes ao dia), glicazida 90 mg (uma vez ao dia), lisinopril 10 mg (uma vez ao dia) e atorvastatina 40 mg (uma vez ao dia). Recorreu ao serviço de urgência do Hospital CUF Porto devido a úlcera no pé direito com cerca de dois meses de evolução, que nos dois dias anteriores tinha vindo a ser acompanhada de dores. Estas apresentavam como fator de alívio a pendência do membro e impediam o sono. o doente negou história de traumatismo e claudicação. Negou também hipertermia ou sudorese. Ao exame objetivo eram palpáveis os pulsos femorais e poplíteos bilateralmente e o pulso pedioso do pé esquerdo. Os pulsos pedioso e tibial posterior do pé direito estavam ausentes. $\bigcirc$ pé direito encontrava-se pálido, marmoreado e com dor ao toque. Verificava-se a presença 


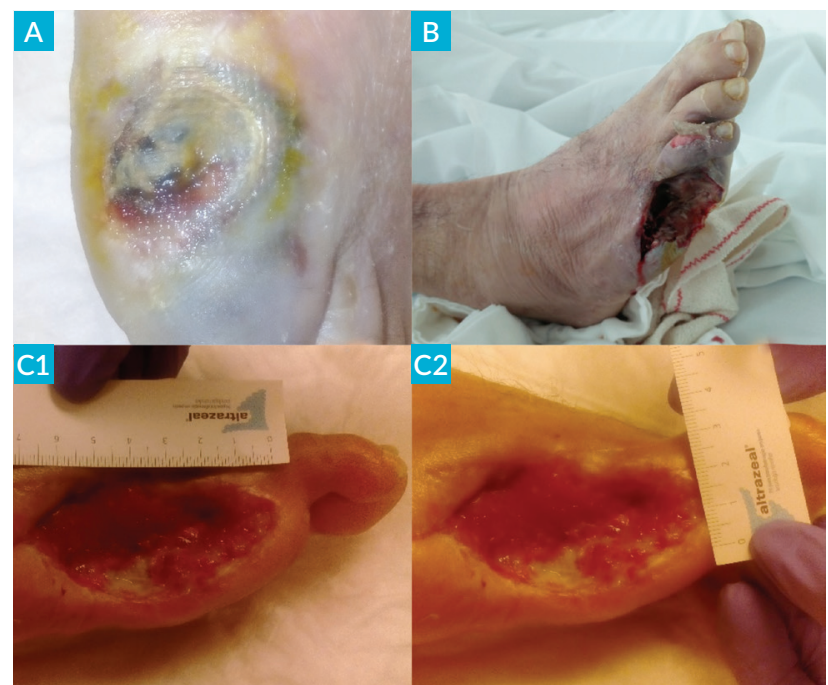

FIGURA 1. A - Úlcera superficial, $1,5 \times 1,5$ cm de dimensão, de bordos regulares, com tecido desvitalizado, localizada no bordo lateral do pé; B - Pós-angioplastia com balão da artéria tibial anterior e amputação aberta do 5º dedo; C1 e C2 - Evolução cicatricial após cirurgias.

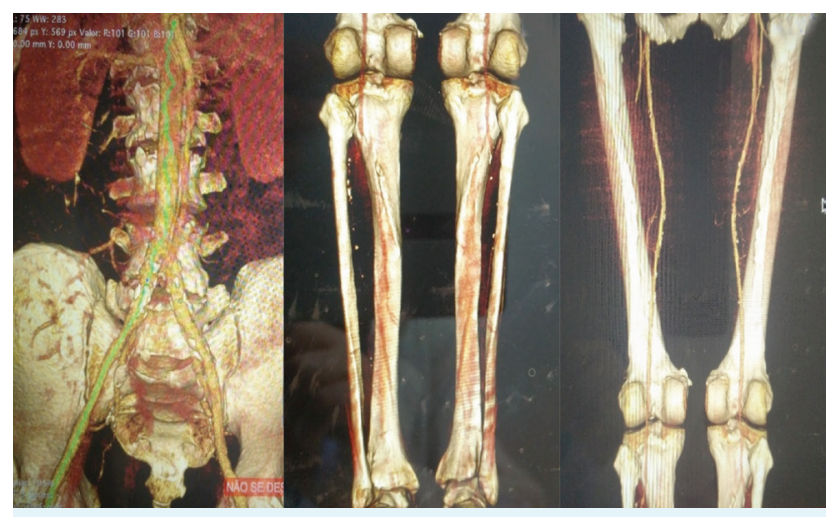

FIGURA 2. AngioTC que revelou normal permeabilidade da aorta, artérias ilíacas, femorais comuns, femorais superficiais e poplíteas e lesões ateroscleróticas oclusivas das artérias tibiais anterior e posterior e peroneal direitas.

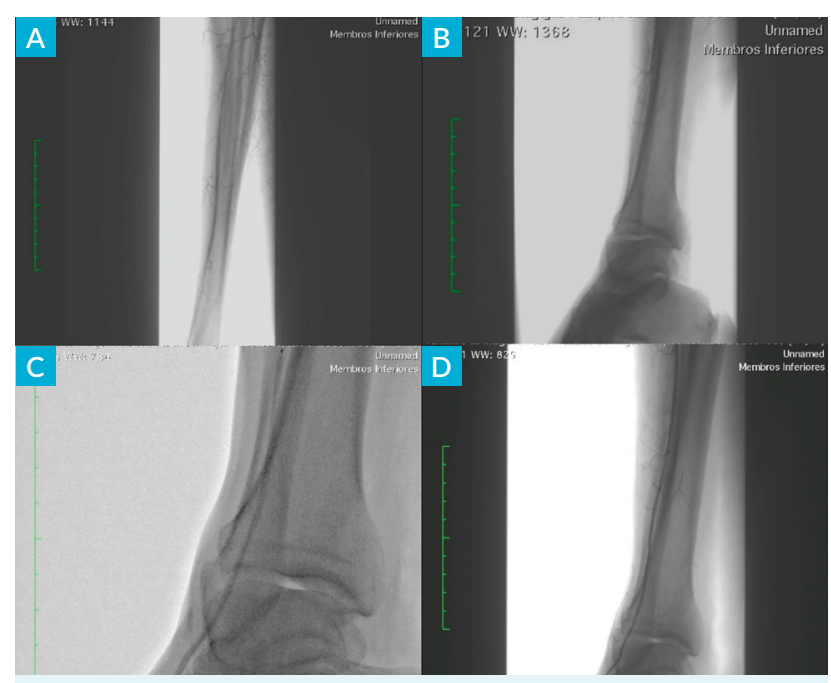

FIGURA 3. A- Múltiplas estenoses da artéria tibial anterior proximalmente, estenoses no tronco tíbio-peroneal, oclusão distal da artéria tibial anterior; B - Oclusão da artéria tibial anterior distalmente; C - Angioplastia com balão da artéria tibial anterior D - Artéria tibial anterior e pediosa após repermeabilização. de uma úlcera superficial, 1,5 × 1,5 cm de dimensão, de bordos regulares, com tecido desvitalizado, localizada no bordo lateral do pé (na base do quinto dedo). Com um estilete era possível tocar osso no fundo da úlcera. Apresentava um IDTB = 100/160 = 0,62. Tinha pressão tibial posterior direita de $100 \mathrm{mmHg}$, tibial anterior direita de $80 \mathrm{mmHg}$ e peroneal direita de $90 \mathrm{mmHg}$.

Diagnosticou-se uma isquemia crónica do membro inferior direito já com uma úlcera infetada no terço distal da face externa do pé (Fig. 1a). Realizou angiotomografia computorizada (angioTC) que revelou normal permeabilidade da aorta, artérias ilíacas, femorais comuns, femorais superficiais e poplíteas e lesões ateroscleróticas oclusivas das artérias tibiais anterior e posterior e peroneal direitas (Fig. 2). Foi internado para controlo de infeção, analgesia e revascularização. Realizou angiografia que confirmou uma obstrução das artérias distais (Fig.s 3a e 3b). Foi realizada uma angioplastia com balão da artéria tibial anterior (Fig. 3c) e amputação aberta do quinto dedo (Fig. 1b). Por má evolução cicatricial e progressão local da destruição tecidular procedeu-se a amputação aberta do quarto e terceiro dedos. Durante o internamento foi medicado com tazobac e vancomicina. Teve alta ao fim de 15 dias. O doente mantém cuidados de penso em regime ambulatório com excelente evolução cicatricial (Fig.s 1c1 e 1c2). Apresenta pulso pedioso direito e deambula de forma autónoma.

\section{DISCUSSÃO}

Este caso clínico ilustra uma das complicações mais frequentes da DM, o pé diabético, num doente de 46 anos, com necessidade de revascularização e amputação minor.

O pé diabético constitui uma das complicações mais frequentes da DM, estimando-se que atinja cerca de 15\% da população diabética em geral e mais de $20 \%$ no grupo de doentes com idade superior a 60 anos. ${ }^{7}$

O pior outcome para um pé diabético é a amputação, em particular a amputação major. As indicações para esta normalmente são múltiplas e as mais citadas são a gangrena e infeção, ocorrendo com frequência de forma simultânea. ${ }^{5}$

A DAOP resulta da obstrução da árvore arterial dos membros inferiores, levando a uma diminuição do aporte sanguíneo necessário aos tecidos durante o exercício ou mesmo em repouso. A necessidade de aporte sanguíneo na presença de uma úlcera é maior devido à necessidade de reparação tecidular, como observado no caso clínico. A gravidade dos sintomas encontra-se depen- 
dente da extensão do processo obstrutivo, mas também da circulação colateral existente. ${ }^{8}$

O desafio na revascularização de pacientes com isquemia crítica, em particular, com envolvimento das artérias abaixo do joelho, é decidir quais as artérias que devem ser repermeabilizadas para alcançar com sucesso o resgate do membro. Apesar de arbitrariamente se recanalizar uma artéria tibial apenas com base na arteriografia, devem ser considerados alguns princípios básicos, tais como o angiossoma. Este delimita, em termos tridimensionais, unidades anatómicas de tecido que são alimentadas por uma dada artéria de origem. No pé estão descritos seis angiossomas, decorrentes da artéria tibial posterior ( $n=3)$, artéria tibial anterior $(n=1)$ e artéria peroneal $(n=2)$. No caso supracitado foi recanalizada a artéria tibial anterior que continua com a artéria pediosa, alimentando o dorso do pé e consequentemente a área da úlcera, ou seja, a área com maior necessidade de aporte sanguíneo. Esta abordagem racional para a revascularização do pé, foi seguida por vários autores que confirmaram a sua relevância para a cicatrização da úlcera e consequente resgate de membros. ${ }^{9}$ Também neste caso, esta estratégia se revelou bem-sucedida, apresentando o doente uma excelente evolução cicatricial, tendo sido evitada uma amputação major. Além da revascularização, um controlo da infeção e cuidados de penso adequados, são fundamentais. ${ }^{10}$

Consideramos o presente caso clínico pertinente dada a prevalência crescente de DM e consequentemente da DAOP. Salienta-se a importância da abordagem multidisciplinar (Endocrinologia, Ortopedia e Cirurgia Vascular), que levam a um correto diagnóstico e à instituição de tratamento precoce adequado das lesões do pé diabético, o que poderá conduzir a uma diminuição da taxa de complicações graves como amputações. ${ }^{7}$

CONFLITOS DE INTERESSE: Os autores declaram não ter qualquer conflito de interesse na realização do presente trabalho.

FONTES DE FINANCIAMENTO: Não houve qualquer fonte de financiamento na realização do presente trabalho.

CONFIDENCIALIDADE DOS DADOS: Os autores declaram ter seguido os protocolos da sua instituição acerca da publicação dos dados de doentes.

PROTEÇÃO DE PESSOAS E ANIMAIS: Os autores declaram que os procedimentos seguidos na elaboração do presente trabalho estão em conformidade com as normas das comissões de investigação clínica e de ética, bem como da declaração de Helsínquia e da Associação Médica Mundial.
CONFLICTS OF INTEREST: The authors declare that they have no conflicts of interest.

FINANCIAL SUPPORT: This work has not received any contribution, grant or scholarship.

CONFIDENTIALITY OF DATA: The authors declare that they have followed the protocols of their work center on the publication of data from patients.

PROTECTION OF HUMAN AND ANIMAL SUBJECTS: The authors declare that the procedures followed were in accordance with the regulations of the relevant clinical research ethics committee and with those of the Code of Ethics of the World Medical Association (Declaration of Helsinki).

\section{REFERÊNCIAS}

1. Observatório Nacional da Diabetes. Diabetes: Factos e Números - O Ano de 2015. Lisboa: Letra Solúvel; 2016.

2. Instituto Nacional de Estatística. Boletim Mensal De Estatística Junho 2017. Lisboa: INE; 2017.

3. Rahaman K, Majdzadeh R, Holakouie Naieni K, Raza O. Knowledge, attitude and practices (KAP) regarding chronic complications of diabetes among patients with type 2 diabetes. Int J Endocrinol Metab. 2017;15:e12555.

4. Lipsky BA; International consensus group on diagnosing and treating the infected diabetic foot. A report from the international consensus on diagnosing and treating the infected diabetic foot. Diabetes Metab Res Rev. 2004;20 Suppl 1:S68-77.

5. Ndosi M, Wright-Hughes A, Brown S, Backhouse M, Lipsky B, Bhogal $M$, et al. Prognosis of the infected diabetic foot ulcer: a 12-month prospective observational study. Diabet Med. 2017 (in press).

6. Gouveia R, Brandão P, Lobo M, Brandão D, Vasconcelos J, Sousa P, et al. Tratamento endovascular de doença arterial obstrutiva abaixo do joelho: existem limites para a revascularização? - Experiência de 5 anos de um centro. Angiol Cir Vasc. 2016; 12:246-51.

7. Horta C, Vilaverde J, Mendes P, Gonçalves I, Serra L, Pinto PS, et al. Avaliação da taxa de amputações - consulta multidisciplinar do pé diabético. Acta Med Port. 2003;16:373-80.

8. Brandão D, Costa C, Mansilha A. Angiogénese e Arteriogénese na Doença Arterial Periférica. Angiol Cir Vasc. 2012;8:53-9.

9. Brandão D, Ferreira J, Mansilha A, Vaz A. Below the knee techniques: now and then. In: Forbes T, editor. Angioplasty, various techniques and challenges in treatment of congenital and acquired vascular stenoses. London: InTech; 2012. p.41-62.

10. Wu J, Wong M, Lo Z, Wong W, Narayanan S, Tan G, et al. A series of 210 peripheral arterial disease below-knee amputations and predictors for subsequent above-knee amputations. Ann Vasc Dis. 2017 (in press). 